PROCEEDINGS OF THE

AMERICAN MATHEMATICAL SOCIETY

Volume 128, Number 8, Pages 2365-2371

S 0002-9939(00)05635-5

Article electronically published on February 25, 2000

\title{
DESYNCHRONIZATION OF LARGE SCALE DELAYED NEURAL NETWORKS
}

\author{
YUMING CHEN, YING SUE HUANG, AND JIANHONG WU
}

(Communicated by Hal L. Smith)

\begin{abstract}
We consider a ring of identical neurons with delayed nearest neighborhood inhibitory interaction. Under general conditions, such a network has a slowly oscillatory synchronous periodic solution which is completely characterized by a scalar delay differential equation with negative feedback. Despite the fact that the slowly oscillatory periodic solution of the scalar equation is stable, we show that the associated synchronous solution is unstable if the size of the network is large.
\end{abstract}

\section{INTRODUCTION}

We consider a ring of identical neurons with delayed nearest neighborhood inhibitory interaction. The dynamics of such a network is described by the following system of delay differential equations

$$
\dot{x}_{i}(t)=-\mu x_{i}(t)-\frac{1}{2}\left[f\left(x_{i-1}(t-r)\right)+f\left(x_{i+1}(t-r)\right)\right],
$$

where $i(\bmod n)$ for a given positive integer $n, \mu$ and $r$ are positive real constants and the activation function $f: \mathbb{R} \rightarrow \mathbb{R}$ is bounded and $C^{1}$-smooth with $f(0)=0$ and $f^{\prime}(x)>0$ for all $x \in \mathbb{R}$. Such a system is a special case of the well-known Hopfield's model for a network of amplifiers (neurons) which, as an electronic device, was designed as a hardware implementation of biological neural networks (Hopfield [6]). The delay was first explicitly introduced by Marcus and Westervelt 9] to account for the finite switching speed of amplifiers. It should be mentioned that the constant $r$ here is not the absolute size of the time lag required for the communication and response among neurons. In fact, system (11) is obtained after some rescaling and reparametrization, and the constant $r$ represents the ratio of the absolute size of the delay over the relaxation time of the system (see, for example, Belair, Campbell and van den Driessche [1, Marcus and Westervelt [9] and Wu [1]). Hence, this constant can be relatively large, and in such a case the dynamics of system (1) can be significantly different from that of the corresponding ordinary differential equation model.

Received by the editors September 19, 1998.

2000 Mathematics Subject Classification. Primary 34K15, 92B20.

Key words and phrases. Synchronization, slow oscillation, delay, neural network.

This research was partially supported by the Natural Sciences and Engineering Research Council of Canada. 
The focus of this paper is the desynchronization of system (1) with large $n$. For system (1), we say that a solution $x=\left(x_{1}, \cdots, x_{n}\right)^{T}:[-r, \infty) \rightarrow \mathbb{R}^{n}$ is synchronous if $x_{1}(t)=\cdots=x_{n}(t)$ for all $t \in[-r, \infty)$, and asynchronous if otherwise. Similarly, we can speak of synchronous or asynchronous states in the phase space $C^{n}=$ $C\left([-r, 0] ; \mathbb{R}^{n}\right)$.

A synchronous solution is completely characterized by the following scalar delay differential equation with negative feedback

$$
\dot{z}(t)=-\mu z(t)-f(z(t-r))
$$

which has been extensively investigated in the literature. In particular, it is shown that for $r$ in a certain range equation (2) has a slowly oscillatory periodic solution. Here and in what follows, a slowly oscillatory periodic solution of equation (2) is a periodic solution $p: \mathbb{R} \rightarrow \mathbb{R}$ of (2) such that distances of consecutive zeros are larger than $r$, and the minimal period $\omega$ is the distance of 3 consecutive zeros. Clearly, $\omega>2 r$. The stability of such a periodic solution of (2) was studied by Chow and Walther [3] (coupled with the technique developed in Ivanov, Lani-Wayda and Walther [7] since $\mu>0$ ) and by Xie [12, 14, 13], to name a few. Such a stability implies the stability of the synchronized periodic solution $p^{s}=(p, \cdots, p)^{T}: \mathbb{R} \rightarrow \mathbb{R}^{n}$ of the network (1) under small synchronous perturbation. This paper, however, shows that the above periodic solution of (1) is always unstable (of course, under asynchronous perturbation) if the network is large. This shows, in terms of the aforementioned stability of the periodic solution $p$ of (2), that the large scale and the delayed inhibitation jointly lead to desynchronization in the considered network of neurons.

\section{MAIN RESULTS}

Let $p: \mathbb{R} \rightarrow \mathbb{R}$ be a slowly oscillatory periodic solution of (2) of the minimal period $\omega>2 r$. Let $p^{s}$ be the corresponding synchronous periodic solution of system (1). By linearizing system (1) around $p^{s}$, we obtain the following linear periodic delay differential system:

$$
\dot{y}_{i}(t)=-\mu y_{i}(t)-\frac{1}{2} f^{\prime}(p(t-r))\left[y_{i-1}(t-r)+y_{i+1}(t-r)\right],
$$

where $i(\bmod n)$

Let $C_{\mathbb{C}}^{n}=C\left([-r, 0] ; \mathbb{C}^{n}\right)$ and let $M_{\mathbb{C}}: C_{\mathbb{C}}^{n} \rightarrow C_{\mathbb{C}}^{n}$ be the monodromy operator of system (B), namely

$$
M_{\mathbb{C}}(\phi)(s)=y^{\phi}(\omega+s) \quad \text { for } s \in[-r, 0], \phi \in C_{\mathbb{C}}^{n},
$$

where $y^{\phi}$ is the solution $y=\left(y_{1}, \cdots, y_{n}\right)^{T}:[-r, \infty) \rightarrow \mathbb{C}^{n}$ of system (3) with $y(s)=\phi(s)$ for $s \in[-r, 0]$. Because $\omega>2 r, M_{\mathbb{C}}$ is linear, continuous and compact, and every point $\lambda \in \sigma\left(M_{\mathbb{C}}\right) \backslash\{0\}$ is an eigenvalue of finite multiplicity and is isolated in $\sigma\left(M_{\mathbb{C}}\right)$. These eigenvalues are called Floquet multipliers of $p^{s}$, which determine the linear stability of the synchronous periodic solution $p^{s}$. See, for example, Diekmann, van Gils, Verduyn Lunel and Walther [4].

Our main result is as follows:

Theorem. Let $p: \mathbb{R} \rightarrow \mathbb{R}$ be a slowly oscillatory periodic solution of (2) and let $p^{s}$ be the corresponding synchronous periodic solution of system (1). Then for large $n$, $p^{s}$ is linearly unstable. More precisely, $p^{s}$ has a real Floquet multiplier larger than 1 . 


\section{THE PROOF AND REMARKS}

Let $\sigma\left(M_{\mathbb{C}}\right)$ be the spectrum of the monodromy operator of system (3). Our goal is to show that $\sigma\left(M_{\mathbb{C}}\right)$ contains a real $\lambda>1$ if $n$ is large. For the sake of simplicity, we let $M_{b}: C_{\mathbb{C}}=C_{\mathbb{C}}^{1} \rightarrow C_{\mathbb{C}}$ denote the linear compact operator given by $\left(M_{b} \varphi\right)(\theta)=u^{\varphi}(\omega+\theta)$ for $\theta \in[-r, 0]$, where $b: \mathbb{R} \rightarrow \mathbb{R}$ is a continuous map with a period $\omega>2 r, u^{\varphi}$ is the solution of

$$
\dot{u}(t)=-\mu u(t)+b(t) u(t-r)
$$

with $\left.u^{\varphi}\right|_{[-r, 0]}=\varphi \in C_{\mathbb{C}}$. Note that $\lambda \in \sigma\left(M_{b}\right) \backslash\{0\}$ if and only if (4) has a nonzero solution $u: \mathbb{R} \rightarrow \mathbb{C}$ such that $u(t+\omega)=\lambda u(t)$ for all $t \in \mathbb{R}$ (see, for example, Hale and Verduyn Lunel [5], Diekmann, van Gils, Verduyn Lunel and Walther 4]).

Let

$$
b_{k}(t)=-f^{\prime}(p(t-r)) \cos \frac{2 k \pi}{n}, k \in\{0,1, \cdots, n-1\} .
$$

We have

Lemma 1. $\bigcup_{k=0}^{n-1} \sigma\left(M_{b_{k}}\right) \backslash\{0\} \subset \sigma\left(M_{\mathbb{C}}\right) \backslash\{0\}$.

Proof. Assume $\lambda \in \sigma\left(M_{b_{k}}\right) \backslash\{0\}$ for some $k \in\{0,1, \cdots, n-1\}$. Then there exists $\psi \in C_{\mathbb{C}} \backslash\{0\}$ such that the solution of (4) with $b=b_{k}$ and $\left.u\right|_{[-r, 0]}=\psi$ satisfies $u(t+\omega)=\lambda u(t)$ for $t \geq-r$. Let $y=\left(y_{1}, \cdots, y_{n}\right)^{T}:[-r, \infty) \rightarrow \mathbb{C}^{n}$ be given by

$$
y_{j}(t)=e^{i \frac{2 \pi}{n} k j} u(t), \quad t \geq-r, j=1, \cdots, n .
$$

Then for $t \geq 0$, we have

$$
\dot{y}_{j}(t)=e^{i \frac{2 \pi}{n} k j}\left[-\mu u(t)+b_{k}(t) u(t-r)\right]=-\mu y_{j}(t)+e^{i \frac{2 \pi}{n} k j} b_{k}(t) u(t-r) .
$$

Note that

$$
\begin{aligned}
y_{j-1}(t-r)+y_{j+1}(t-r) & =\left[e^{i \frac{2 \pi}{n} k(j-1)}+e^{i \frac{2 \pi}{n} k(j+1)}\right] u(t-r) \\
& =2 \cos \frac{2 \pi k}{n} e^{i \frac{2 \pi}{n} k j} u(t-r) .
\end{aligned}
$$

Therefore for $t \geq 0$, we have

$$
\dot{y}_{j}(t)=-\mu y_{j}(t)-\frac{1}{2} f^{\prime}(p(t-r))\left[y_{j-1}(t-r)+y_{j+1}(t-r)\right] .
$$

As $\left.y\right|_{[-r, 0]}=\left(e^{i \frac{2 \pi}{n} k}, e^{i \frac{2 \pi}{n}(2 k)}, \cdots, e^{i \frac{2 \pi}{n}(n k)}\right)^{T} \psi \in C_{\mathbb{C}}^{n} \backslash\{0\}$ and

$$
y_{j}(t+\omega)=e^{i \frac{2 \pi}{n} k j} u(t+\omega)=\lambda e^{i \frac{2 \pi}{n} k j} u(t)=\lambda y_{j}(t), t \geq-r, j=1, \cdots, n,
$$

we conclude that $\lambda \in \sigma\left(M_{\mathbb{C}}\right)$. This completes the proof.

The next result concerns the leading eigenvalue of $M_{-b_{0}}$, where $-b_{0}=$ $f^{\prime}(p(t-r))$. Recall that $\omega>2 r$.

Lemma 2. There exists a real simple eigenvalue $\lambda>1$ of $M_{-b_{0}}$ with an associated eigenvector $\phi \in C^{+}:=\{\phi \in C([-r, 0] ; \mathbb{R}) ; \phi(s)>0$ for $s \in[-r, 0]\}$.

Proof. Due to the increasing property of $f$, it is easy to show that $\left.M_{-b_{0}}\right|_{C}: C=$ $C^{1} \rightarrow C$ is a positive linear operator. Therefore, the existence of a real eigenvalue $\lambda>0$ of $M_{-b_{0}}$ associated with an eigenvector $\phi \in C^{+}$is an immediate consequence of the Krein-Rutman theorem (see, for example, Smith [10]). So it suffices to show that $\lambda>1$. 
We are going to use the following simple coupling technique (used in the work of Chen, Krisztin and Wu [2]): $(X, Y)^{T}: \mathbb{R} \rightarrow \mathbb{C}^{2}$ is a solution of the decoupled system

$$
\dot{X}(t)=-\mu X(t)+b_{0}(t) X(t-r)
$$

and

$$
\dot{Y}(t)=-\mu Y(t)-b_{0}(t) Y(t-r)
$$

if and only if $(U, V)^{T}: \mathbb{R} \rightarrow \mathbb{C}^{2}$ given by

$$
U(t)=X(t)+Y(t), V(t)=-X(t)+Y(t)
$$

is a solution of the coupled system

$$
\left\{\begin{array}{l}
\dot{U}(t)=-\mu U(t)-b_{0}(t) V(t-r) \\
\dot{V}(t)=-\mu V(t)-b_{0}(t) U(t-r)
\end{array}\right.
$$

In particular, $(\dot{p},-\dot{p})^{T}: \mathbb{R} \rightarrow \mathbb{R}^{2}$ is an $\omega$-periodic solution of (9).

Let $F: C^{2} \rightarrow C^{2}$ be the (real) monodromy operator of system (9). Namely,

$$
F(\psi)(\theta)=\left(U^{\psi}(\omega+\theta), V^{\psi}(\omega+\theta)\right)^{T}, \theta \in[-r, 0], \psi \in C^{2},
$$

where $\left(U^{\psi}, V^{\psi}\right)^{T}$ is the solution of (9) with $\left.\left(U^{\psi}, V^{\psi}\right)^{T}\right|_{[-r, 0]}=\psi$. Let

$$
K^{2}=\left\{\psi=\left(\psi_{1}, \psi_{2}\right)^{T} \in C^{2} ; \psi_{i}(s) \geq 0 \text { for all } i=1,2 \text { and } s \in[-r, 0]\right\} .
$$

It is easy to verify, due to $\omega>r$ and $b_{0}(t)<0$ for all $t \in \mathbb{R}$, the following order preserving property

$$
F\left(K^{2}\right) \subset K^{2}, \quad F^{2}\left(K^{2} \backslash\{0\}\right) \subset i n t K^{2} .
$$

We have noticed that $(\phi, \phi)^{T} \in i n t K^{2}$ is an eigenvector of $F$ associated with some real $\lambda>0$. We now follow the trick in Krisztin, Walther and $\mathrm{Wu} 8$ to show that $\lambda>1$. If $\lambda<1$, then $(\phi, \phi)^{T}+\varepsilon\left(\dot{p}_{0},-\dot{p}_{0}\right)^{T} \in K^{2}$ for small $\varepsilon>0$ and thus

$$
F^{j}\left((\phi, \phi)^{T}+\varepsilon\left(\dot{p}_{0},-\dot{p}_{0}\right)^{T}\right)=\lambda^{j}(\phi, \phi)^{T}+\varepsilon\left(\dot{p}_{0},-\dot{p}_{0}\right)^{T} \rightarrow \varepsilon\left(\dot{p}_{0},-\dot{p}_{0}\right)^{T} \in C^{2} \backslash K^{2}
$$

as $j \rightarrow \infty$. Therefore, for large $j$, we have $F^{j}\left((\phi, \phi)^{T}+\varepsilon\left(\dot{p}_{0},-\dot{p}_{0}\right)^{T}\right) \notin K^{2}$, a contradiction to (10). We now exclude the case where $\lambda=1$ by contradiction. Assume that $\lambda=1$. There must be $\tau>0$ such that $(\phi, \phi)^{T}+\tau\left(\dot{p}_{0},-\dot{p}_{0}\right)^{T} \in$ $C^{2} \backslash K^{2}$. Therefore, there exists $s \in(0, \tau)$ such that $(\phi, \phi)^{T}+\beta\left(\dot{p}_{0},-\dot{p}_{0}\right)^{T} \in$ int $K^{2}$ for $\beta \in(0, s)$ and $(\phi, \phi)^{T}+s\left(\dot{p}_{0},-\dot{p}_{0}\right)^{T} \in K^{2} \backslash$ int $K^{2}$. We clearly have $(\phi, \phi)^{T}+s\left(\dot{p}_{0},-\dot{p}_{0}\right)^{T} \neq 0$ since $(\phi, \phi)^{T} \in$ int $K^{2}$ and $s\left(-\dot{p}_{0},-\dot{p}_{0}\right)^{T} \notin K^{2}$. Hence, $F^{2}\left((\phi, \phi)^{T}+s\left(\dot{p}_{0},-\dot{p}_{0}\right)^{T}\right)=(\phi, \phi)^{T}+s\left(\dot{p}_{0},-\dot{p}_{0}\right)^{T} \in K^{2} \backslash i n t K^{2}$, a contradiction to (10). This shows $\lambda>1$, completing the proof.

The following result shows the persistence of the monodromy operator under small perturbation. For a general result regarding the persistence of the spectra of Poincaré-type mappings associated with delay differential equations, see Ivanov, Lani-Wayda and Walther [7].

Lemma 3. Assume that $c_{k}: \mathbb{R} \rightarrow \mathbb{R}$ are continuous and $\omega$-periodic and $c_{k}(t) \rightarrow$ $b_{0}(t)$ as $k \rightarrow \infty$, uniformly for $t \in \mathbb{R}$. Then for large $k, M_{-c_{k}}$ has a real eigenvalue $\lambda_{k}>1$ with an associated eigenvector $\phi_{k} \in C^{+}$. 
Proof. Let $\lambda$ be given in Lemma 2 and define $\alpha=\frac{1}{\omega} \ln \lambda$. Then there exists a positive $\omega$-periodic $C^{1}$-smooth map $q: \mathbb{R} \rightarrow \mathbb{R}$ such that $e^{\alpha t} q(t)$ is a corresponding (characteristic) solution of (4) with $b(t)=-b_{0}(t)$ for all $t \in \mathbb{R}$. Then

$$
\dot{q}(t)+(\alpha+\mu) q(t)+b_{0}(t) e^{-\alpha r} q(t-r)=0, \quad t \in \mathbb{R} .
$$

Let $H_{\omega}^{0}$ denote the Banach space of $\omega$-periodic continuous real mappings equipped with the super-norm $|\cdot|_{H_{\omega}^{0}}$ and let $H_{\omega}^{1}=\left\{w \in H_{\omega}^{0} ; w\right.$ is $C^{1}$-smooth and $\left.|\dot{w}|_{H_{\omega}^{0}}<\infty\right\}$. Then $H_{\omega}^{1}$, equipped with the norm $|w|_{H_{\omega}^{1}}=|w|_{H_{\omega}^{0}}+|\dot{w}|_{H_{\omega}^{0}}$ for $w \in H_{\omega}^{1}$ is a Banach space. Define $T=\left(T_{1}, T_{2}\right): H_{\omega}^{1} \times \mathbb{R} \times H_{\omega}^{0} \rightarrow H_{\omega}^{0} \times \mathbb{R}$ by

$$
T_{1}(w, \nu, b)=\dot{w}(t)+(\mu+\nu) w(t)+b(t) e^{-\nu r} w(t-r)
$$

and

$$
T_{2}(w, \nu, b)=\frac{1}{\omega} \int_{0}^{\omega} w(t) d t-\frac{1}{\omega} \int_{0}^{\omega} q(t) d t
$$

where $(w, \nu, b) \in H_{\omega}^{1} \times \mathbb{R} \times H_{\omega}^{0}$. Then $T\left(q, \alpha, b_{0}\right)=0$. We will show that $\left.D_{(w, \nu)} T\right|_{\left(q, \alpha, b_{0}\right)}$ is bijective.

First of all, we note that

$$
\begin{aligned}
\left.D_{(w, \nu)} T\right|_{\left(q, \alpha, b_{0}\right)}(u, \Gamma)= & \left(\dot{u}(t)+(\alpha+\mu) u(t)+b_{0}(t) e^{-\alpha r} u(t-r)\right. \\
& \left.+\Gamma\left(q(t)-b_{0}(t) e^{-\alpha r} r q(t-r)\right), \frac{1}{\omega} \int_{0}^{\omega} u(t) d t\right) .
\end{aligned}
$$

We claim that $\left.\operatorname{Ker} D_{(w, \nu)} T\right|_{\left(q, \alpha, b_{0}\right)}=(0,0)$. To verify this claim, we suppose there exists $(u, \Gamma)$ such that

$$
\left.D_{(w, \nu)} T\right|_{\left(q, \alpha, b_{0}\right)}(u, \Gamma)=(0,0) .
$$

Then

$$
\dot{u}(t)+(\alpha+\mu) u(t)+b_{0}(t) e^{-\alpha r} u(t-r)+\Gamma\left[q(t)-b_{0}(t) e^{-\alpha r} r q(t-r)\right]=0
$$

and

$$
\frac{1}{\omega} \int_{0}^{\omega} u(t) d t=0
$$

If $\Gamma \neq 0$, then $u \neq 0$ and $e^{\alpha t} q(t)$ and $e^{\alpha t}\left[t q(t)+\Gamma^{-1} u(t)\right]$ are both solutions of equation (4) with $b=-b_{0}$, a contradiction to the simplicity of the eigenvalue $\lambda=e^{\omega \alpha}$ of $M_{-b_{0}}$. If $\Gamma=0$, then

$$
\dot{u}(t)+(\mu+\alpha) u(t)+b_{0}(t) e^{-\alpha r} u(t-r)=0 .
$$

Since $\lambda$ as an eigenvalue of $M_{-b_{0}}$ is simple, we have that $u=c q$ for some constant c. But $\frac{1}{\omega} \int_{0}^{\omega} u(t) d t=0$ and $\int_{0}^{\omega} q(t) d t>0$. Therefore, $c=0$ which gives $u=0$.

Next, we show that $\left.D_{(w, \nu)} T\right|_{\left(q, \alpha, b_{0}\right)}$ is surjective. Since $\lambda$ as an eigenvalue of $M_{-b_{0}}$ is simple, the space $\left\{w \in H_{\omega}^{1} ; T_{1}\left(w, \alpha, b_{0}\right)=0\right\}$ is one-dimensional. So

$$
\operatorname{dim} \operatorname{Ker}\left(D_{w} T_{1}\right)=\operatorname{codim}\left(\text { Range } D_{w} T_{1}\right)=1 .
$$

If $\left.D_{(w, \nu)} T_{1}\right|_{\left(q, \alpha, b_{0}\right)}$ is not surjective, then

$$
\operatorname{Range}\left(D_{w} T_{1}\right)+\operatorname{Range}\left(D_{\nu} T_{1}\right) \neq H_{\omega}^{0} .
$$

Therefore,

$$
\operatorname{Range}\left(D_{\nu} T_{1}\right) \subset \operatorname{Range}\left(D_{w} T_{1}\right) .
$$


Thus, there exists a function $u \in H_{\omega}^{1}$ such that $\dot{u}(t)+(\mu+\alpha) u(t)+b_{0}(t) e^{-\alpha r} u(t-r)=$ $-\left[q(t)-b_{0}(t) e^{-\alpha r} r q(t-r)\right]$, which implies that equation (4) has a characteristic solution of form $e^{\alpha t}[t q(t)+u(t)]$, and thus the multiplicity of $\lambda$ as an eigenvalue of $M_{-b_{0}}$ is at least two, a contradiction.

Given any number $m \in \mathbb{R}$ and $\gamma \in H_{\omega}^{0}$, from the above discussion there exists $(u, \Gamma) \in H_{\omega}^{0} \times \mathbb{R}$ such that

$$
\left.D_{(w, \nu)} T_{1}\right|_{\left(q, \alpha, b_{0}\right)}(u, \Gamma)=\gamma .
$$

Since for any constant $k,(u+k q, \Gamma)$ is also a solution of the above equation, we can select $k$ such that $\frac{1}{\omega} \int_{0}^{\omega}(u(t)+k q(t)) d t=m$. This shows the surjectivity of $\left.D_{(w, \nu)} T\right|_{\left(q, \alpha, b_{0}\right)}$.

Then we can use the implicit function theorem to solve equation

$$
T(w, \nu, b)=0 .
$$

In particular, for any $\varepsilon>0$, there exists an open neighborhood $U_{b_{0}, \varepsilon}$ in $H_{\omega}^{0}$ of $b_{0}$ such that for every $b \in U_{b_{0}, \varepsilon}$ the operator equation (13) has a unique solution $\left(q_{b}, \alpha_{b}\right) \in H_{\omega}^{1} \times \mathbb{R}$. One can see that when $\varepsilon$ is small enough, $\alpha_{b}>0$ and $q_{b}(t)>0$ for all $t \in \mathbb{R}$. This completes the proof.

We can now prove the main result:

Proof of the Main Theorem. For $k=\left[\frac{n}{2}\right]$,

$$
\lim _{n \rightarrow \infty} \cos \frac{2 \pi k}{n}=-1 \text {. }
$$

Therefore, $b_{\left[\frac{n}{2}\right]}(t) \rightarrow-b_{0}(t)$ as $n \rightarrow \infty$, uniformly for $t \in \mathbb{R}$. Thus the theorem follows from Lemma 3 .

Remark. The above argument applies to systems with arbitrary even $n$. So synchronous periodic solutions of network (11) arising from the slowly oscillatory periodic solutions of (2) are always unstable for the network (1) with either large $n$ or arbitrary even $n$. However, these synchronous periodic solutions can be stable under possible perturbations if $n$ is odd and small. Examples of this type will be given in the case study of Wu, Faria and Huang [15].

\section{REFERENCES}

[1] J. Bélair, S. A. Campbell and P. van den Driessche, Frustration, stability, and delayinduced oscillations in a neural network model. SIAM J. Appl. Math. 56 (1996), 245-255. MR 96j:92003

[2] Y. Chen, J. Wu and T. Krisztin, Connecting orbits from synchronous periodic solutions to phase-locked periodic solutions in a delay differential system, to appear in Journal of Differential Equations, 1999.

[3] S. N. Chow and H. O. Walther, Characteristic multipliers and stability of symmetric periodic solutions of $\dot{x}(t)=g(x(t-1))$, Trans. Amer. Math. Soc. 307 (1988), 124-142. MR 89d:34115

[4] O. Diekmann, S. A. van Gils, S. M. Verduyn Lunel and H.-O. Walther, Delay Equations, Functional, Complex, and Nonlinear Analysis, Springer-Verlag, New York, 1995. MR 97a:34001

[5] J. K. Hale and S. M. Verduyn Lunel, Introduction to Functional Differential Equations, Applied Mathematical Sciences, Vol. 99, Springer-Verlag, New York, 1993. MR 94m:34169

[6] J. J. Hopfield, Neurons with graded response have collective computational properties like two-stage neurons, Proc. Nat. Acad. Sci. U.S.A. 81 (1984), 3088-3092.

[7] A. Ivanov, B. Lani-Wayda and H. O. Walther, Unstable hyperbolic periodic solutions of differential delay equations, World Sci. Ser. Appl. Anal. 1 (1992), 301-316. MR 93h:34125 
[8] T. Krisztin, H. O. Walther and J. Wu, Shape, Smoothness and Invariant Stratification of an Attracting Set for Delayed Monotone Positive Feedback, Fields Institute Monographs, Vol. 11, American Mathematical Society, Providence, 1999.

[9] C. M. Marcus and R. M. Westervelt, Stability of analog neural networks with delay. Phys. Rev. A (3) 39 (1989), 347-359. MR 89m:92025

[10] H. L. Smith, Monotone Dynamical Systems, An Introduction to the Theory of Competitive and Cooperative Systems, Mathematical Surveys and Monographs, Vol. 41, Amer. Math. Soc., Providence, RI, 1995. MR 96c:34002

[11] J. Wu, Symmetric functional-differential equations and neural networks with memory. Trans. Amer. Math. Soc. 350 (1998), 4799-4838. MR 99i:34104

[12] X. W. Xie, Uniqueness and stability of slowly oscillating periodic solutions of delay equations with bounded nonlinearity, J. Dyna. Diff. Eqns. 3 (1991), 515-540. MR 93b:34093

[13] X. W. Xie, Uniqueness and stability of slowly oscillating periodic solutions of delay equations with unbounded nonlinearity, J. Diff. Eqns. 103 (1993), 350-374. MR 94c:34115

[14] X. W. Xie, The multiplier equation and its application to S-solutions of a delay equation, J. Diff. Eqns. 102 (1992), 259-280. MR 93f:34110

[15] J. Wu, T. Faria and Y. S. Huang, Absolute synchronization and stable phase-locking in a network of neurons with memory, Mathematical and Computer Modeling 30 (1999), 117-138.

Department of Mathematics and Statistics, York University, Toronto, Ontario, CANADA M3J 1P3

E-mail address: yumingc@mathstat.yorku.ca

Department of Mathematics, Pace University, Pleasantville, New York 10570

E-mail address: huang@risc.dac.pace.edu

Department of Mathematics and Statistics, York University, Toronto, Ontario, CANADA M3J 1P3

E-mail address: wujh@mathstat.yorku.ca 